\title{
Design, Construction, and Calibration of a Temperature Monitoring System for Resistance Standards
}

\author{
Daniel Paseltiner ${ }^{1}$, Shamith Payagala ${ }^{2}$, and Dean Jarrett ${ }^{2}$ \\ ${ }^{1}$ Bates College, \\ Lewiston, ME 04240, USA \\ ${ }^{2}$ National Institute of Standards and Technology, \\ Gaithersburg, MD 20899, USA
}

dpaselti@bates.edu

shamith.payagala@nist.gov

dean.jarrett@nist.gov

\begin{abstract}
We present the design, construction, calibration, and software development of a temperature monitoring system for resistance standards. The system supports 19 temperature probes. Over the range $295.15 \mathrm{~K}$ to $299.15 \mathrm{~K}\left(22{ }^{\circ} \mathrm{C}\right.$ to $\left.26{ }^{\circ} \mathrm{C}\right)$, we report an expanded uncertainty $(k=2)$ of $9 \mathrm{mK}$. With the addition of a calibrated standard reference thermometer and a programmable oil bath, the system was used to automatically calibrate the temperature probes over this $4 \mathrm{~K}$ range. In continuous operation, this system supplies a constant current to thermistor temperature probes and a reference resistor, and it measures the voltage across them. The ratio between each of the probe voltages and the reference voltage is multiplied by the reference resistance to determine the resistance of each probe. To reduce systematic errors, voltage measurements are taken with the current running in alternating directions. Finally, using the Steinhart-Hart model, the probe resistances are converted to their corresponding temperatures and recorded to a secure network drive. If a probe reads a temperature outside of the desired temperature range for its location, an email alert is sent to all the staff who work in the laboratory. An additional message will be sent to facility services if the probe is measuring the room temperature in the laboratory. The system was developed for the NIST resistance laboratory, but it could easily be duplicated for use in any laboratory environment where continuous temperature monitoring in multiple locations with expanded uncertainty $(k=2)$ of 9 mK is needed.
\end{abstract}

Key words: resistance; temperature; thermistor; uncertainty; voltage.

Accepted: September 29, 2017

Published: December 18, 2017

https://doi.org/10.6028/jres.122.045

\section{Introduction}

The National Institute of Standards and Technology (NIST) Metrology of the Ohm Project is responsible for maintaining resistance standards and providing resistance calibrations to support the scientific and industrial foundations of the U.S. economy, as well as continuing to develop new resistance measurement techniques with greater accuracy. The expanded uncertainties $(k=2)$ associated with these calibrations range from $0.04 \mu \Omega / \Omega$ to $100 \mu \Omega / \Omega$ over 20 decades of resistance measurement. When measuring resistance to these uncertainty levels, temperature variations, even on the order of $20 \mathrm{mK}$, can have a significant impact, as shown in Fig. 1. In light of this, the resistance standards are measured in temperature-controlled oil and air baths. Ten individual resistance measurement systems monitor temperature during resistance calibration, but no system previously existed to continuously monitor the temperature of these critical locations and provide notification if they have drifted outside the desired temperature ranges $[1,2]$. 


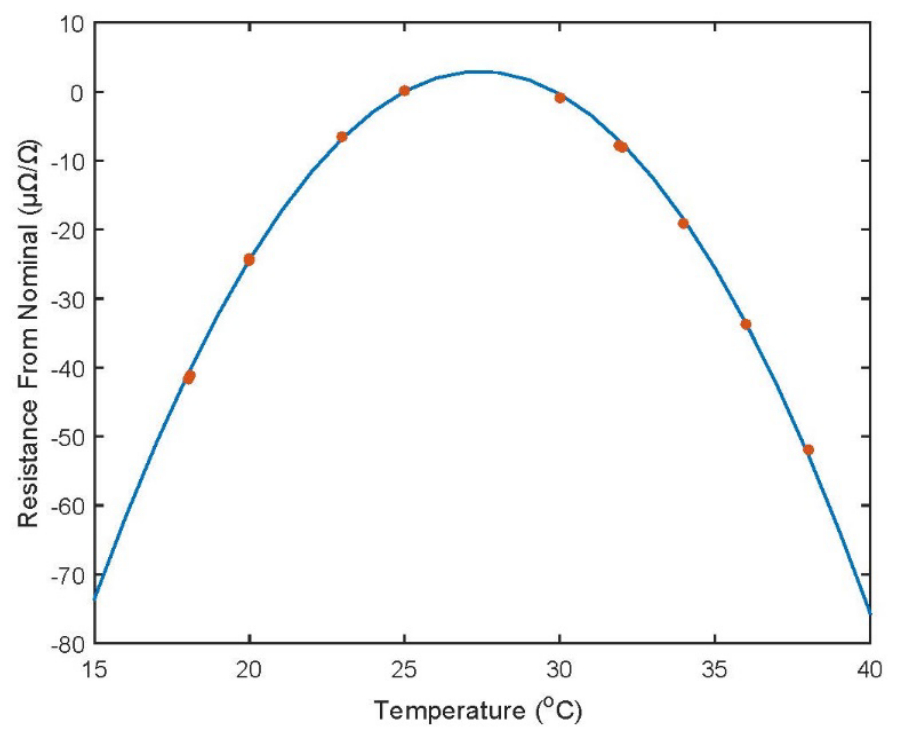

Fig. 1. Temperature dependence of a Thomas $1 \Omega$ standard resistor.

This new infrastructure is designed to alert staff of failure of the building's climate controls or an individual bath, which previously could have taken an extended period of time to discover. Additionally, the system allows continuous tracking of the bath(s) temperatures in the case of such an event. To better manage temperature problems in the resistance laboratory, we chose to design and install a temperature monitoring system capable of:

1. continuous operation,

2. data logging,

3. monitoring up to 20 locations,

4. achieving a $50 \mathrm{mK}$ resolution, and

5. sending email alerts.

Continuous operation and data logging are required because we desired a complete log of bath and laboratory temperature. Currently, there is a need to monitor the temperature in 15 baths as well as 3 locations in laboratory, so a capacity to support 20 probes meets present needs and allows for future expansion. Individual resistance measurement systems are used to record temperatures that allow precise temperature corrections to the value of standard resistors, and to maintain fine temperature control as required, from $\pm 25 \mathrm{mK}$ down to $\pm 3 \mathrm{mK}$. The main purpose of the temperature monitoring system is to alert the laboratory staff of a bath control failure or a heating, ventilation, and air conditioning (HVAC) failure. Therefore, if the system is going to identify when a bath's temperature controller has failed, it must be able to achieve a $50 \mathrm{mK}$ resolution, meaning that the uncertainty of its temperature measurements must be much less than $50 \mathrm{mK}$. Finally, email notifications are a required feature so that the laboratory personnel may be alerted to conditions in the laboratory even when not physically present.

\section{Theory}

Negative temperature coefficient (NTC) thermistors are resistors made from semiconducting elements that are designed to have a predictable dependence on temperature. As temperature increases, more charge carriers become available, causing a decrease in resistance. This allows NTC thermistors to be used as thermometers because their change in resistance is inversely related to temperature [3]. In principle, all that is required to use a thermistor to measure temperature is to measure its resistance once the resistancetemperature curve has been determined. Direct-current resistance measurements are performed with low applied current to avoid self-heating in the thermistor, which is clearly undesirable. To avoid this issue, our system measures the voltage drop across a thermistor probe, which in combination with the voltage drop 
across a resistance standard in series with the probe, can be used to calculate its resistance. These measurements are performed in a four-terminal configuration, where a constant current is supplied across the voltage leads, and the sense leads are used to measure voltage [4]. Figure 2 shows the resistance standard and the thermistor probes connected in series with the constant-current source.

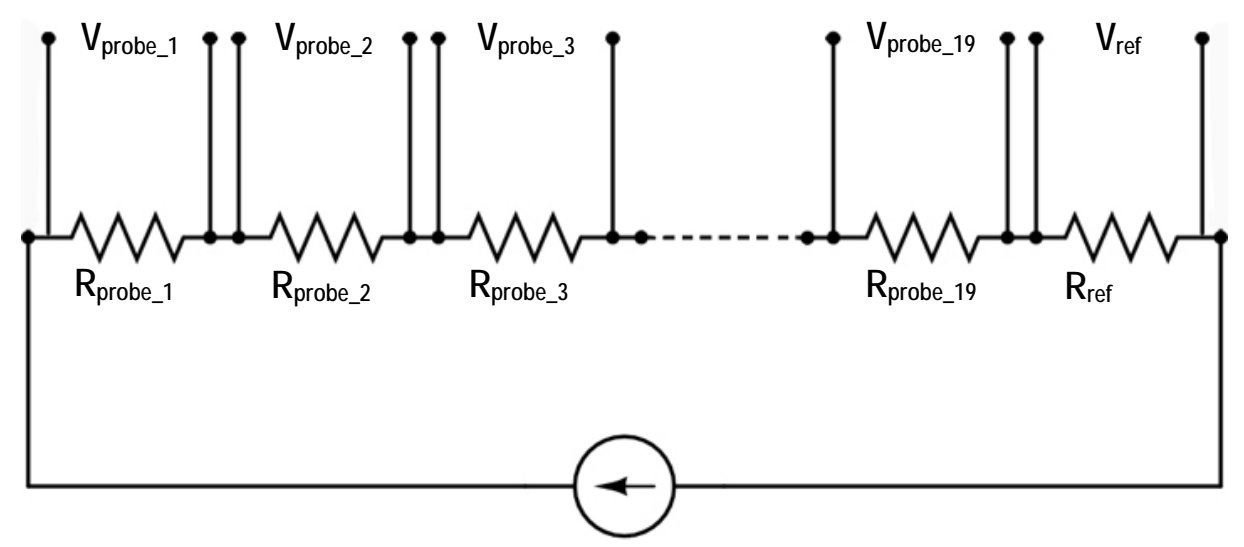

Fig. 2. A voltage divider formed from the probes in series with a reference resistor, driven by a constant-current source.

From Ohm's law, we can write that

$$
\frac{V_{\text {probe }}}{R_{\text {probe }}}=\frac{V_{\text {ref }}}{R_{\text {ref }}}=I
$$

From here, $R_{\text {probe }}$ can be written in terms of $V_{\text {probe }}, V_{\text {ref, }}$ and $R_{\text {ref }}$ as

$$
R_{\text {probe }}=\frac{V_{\text {probe }} R_{\text {ref }}}{V_{\text {ref }}} .
$$

Determining $R_{\text {probe }}$ depends only on measuring $V_{\text {probe }}$ and $V_{\text {ref, }}$ given that $R_{\text {ref }}$ is known. The current is reversed after each set of $V_{\text {probe }}$ and $V_{\text {ref }}$ measurements is made to determine $R_{\text {probe }}$ for both polarities and to cancel the effect of thermal electromotive forces. In this way, we have reduced the temperature measurement to a voltage measurement that can be easily and precisely performed by many commercially available instruments. Adding more temperature probes to the system can be accomplished by placing additional probes in series with the reference. Our system has the capacity to place 19 probes in series with the reference resistor.

\section{Instrumentation}

To implement the measurement technique discussed in the previous section, we assembled the instrumentation shown in Fig. 3. The system is centered around the 20 channel junction box, designed and built at NIST, into which all of the probes and the reference resistor are connected. One channel is dedicated to the reference resistor, leaving 19 channels for the thermistor probes. A constant-current source supplies a $10 \mu \mathrm{A}$ current to all of the channels of the junction box in series. Voltage measurements of the probes and the reference resistor are made using an $8 \frac{1}{2} 2$ digit digital voltmeter (DVM). The channel to be measured is connected to the DVM using a standard cell scanner for switching. The final component of the system is a current reverser, also designed and built at NIST, that allows voltage measurements to be made with the applied direct current in alternating directions, which reduces systematic error. A general purpose interface bus (GPIB) provides the system control to the DVM and scanner. The external output of the DVM controls the current reverser. Measurement, current, and control paths are shown in Fig. 3. 
The thermistor probes used for this system have a nominal resistance of $30 \mathrm{k} \Omega$ at $25{ }^{\circ} \mathrm{C}$ and a typical temperature coefficient of $-130 \Omega /{ }^{\circ} \mathrm{C}$ at $25^{\circ} \mathrm{C}$. The thermistor probes are package in stainless-steel sheaths, and the manufacturer specification is $\pm 0.15^{\circ} \mathrm{C}$ accuracy and interchangeability without calibration. The maximum current for these thermistor probes is $625 \mu \mathrm{A}$, so self-heating at $10 \mu \mathrm{A}$ is negligible. The reference resistor used is a $10 \mathrm{k} \Omega$ standard resistor placed in an oil bath at $25.000 \pm 0.001{ }^{\circ} \mathrm{C}$.

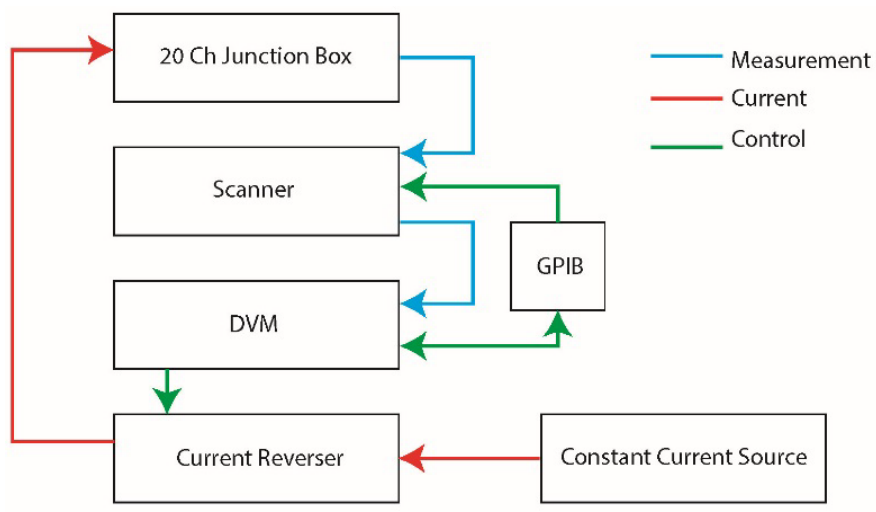

Fig. 3. Block diagram of instrumentation used to measure the thermistor probes.

\subsection{Theory of Operation}

Measuring the resistance of a probe requires the system to measure the voltage across the probe in question and across the reference resistor. To do this, the scanner first connects the probe's channel to the DVM, which then makes five voltage measurements. The external output of the DVM then triggers the current reverser to change the direction of the current with a square pulse. The DVM then makes five more measurements of the probe voltage. The scanner then connects the reference resistor to the DVM, and the same process is used to measure its voltage. The average of each of two sets of ten measurements is then used, along with the resistance of the reference resistor, to compute $R_{\text {probe }}$ using Eq. (2). The required instrument control and data acquisition are provided over a GPIB by a computer running a LabVIEW script. $^{1}$

\subsection{Current Reversal}

To ensure the greatest accuracy possible, it is necessary to make voltage measurements with current running in alternating directions. To incorporate this into the system, an automated reversal of the direction of the current was required. We chose to realize this with the circuit shown in Fig. 4. Here, a D-type flip flop is used as a divide-by-two counter by introducing a feedback loop from $\bar{Q}$ to $D$. The clock is driven by the external output of the DVM, and the output $Q$ is sent to the control pin of a MAXIM 4526 phase reversal integrated circuit [5]. The result is that whenever the clock sees a rising edge from the DVM, the logic value of $Q$ is switched, causing the MAXIM 4526 to change state, thereby reversing the direction of current.

\footnotetext{
${ }^{1}$ Certain commercial equipment, instruments, or materials are identified in this paper in order to specify the experimental procedure adequately. Such identification does not imply recommendation or endorsement by the National Institute of Standards and Technology, nor does it imply that the materials or equipment identified are necessarily the best available for the purpose.
} 


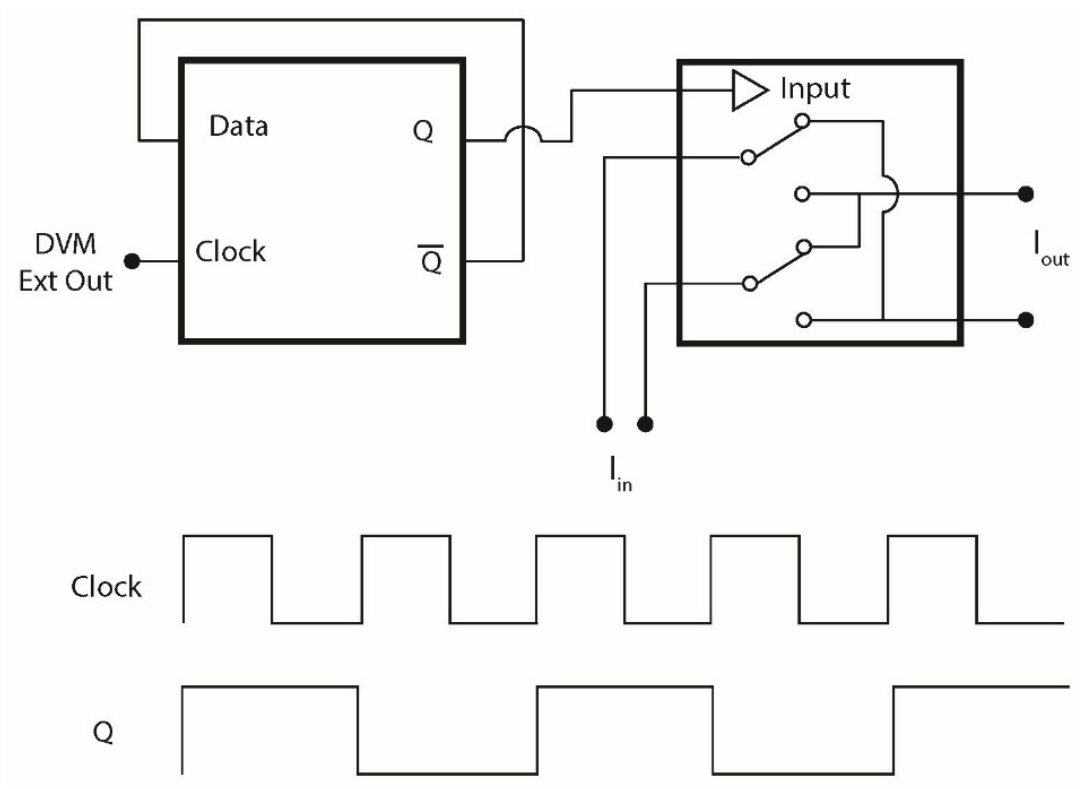

Fig. 4. Current reversal circuit.

\section{Calibration}

We have established how the system will measure the resistance of a probe, but not how to convert these resistances to temperature. Mapping resistances to their corresponding temperatures requires a calibration between the two quantities. The standard method for calibrating thermistors is to use the Steinhart-Hart equation [6, 7],

$$
\frac{1}{T}=A+B \ln R+\mathrm{C} \ln ^{2} R+\mathrm{D} \ln ^{3} R,
$$

which gives temperature $T$ as a function of resistance $R$ of the thermistor, and the four so-called SteinhartHart coefficients $A, B, C$, and $D$. If these coefficients are known, then the temperature associated with any resistance can be calculated.

To determine the Steinhart-Hart coefficients, two additional components are required, an oil bath and a calibrated temperature probe. The oil bath is used to hold the probes to be calibrated at fixed temperatures. The calibrated probe is used as a temperature standard to accurately measure the stable temperatures provided by the bath. This allows for probe resistance to be measured as a function of temperature by varying the set point of the oil bath. Since the air and oil baths for resistance calibration are held at $23^{\circ} \mathrm{C}$ and $25^{\circ} \mathrm{C}$, respectively, we chose to calibrate the probes over a $22^{\circ} \mathrm{C}$ to $26^{\circ} \mathrm{C}$ interval. Resistance measurements were made in whole degree increments of temperature over this range, using the same measurement technique as described earlier. A linear least squares fit was then used to fit Eq. (3) to the five calibration points to determine the Steinhart-Hart coefficients, as shown in Fig. 5. In practice, $C$ is small when compared to the other coefficients, and in most cases, this makes the $C \ln ^{2} R$ term negligible. In light of this, we chose to ignore the contribution of this term and only determine $A, B$, and $D$ from the fit. For this narrow temperature range of $4{ }^{\circ} \mathrm{C}$, the value of $D \ln ^{3} R$ term could have been ignored as well, but it was kept in the analysis to permit accurate curve fitting over wider temperature ranges for future applications with the same software and system. 


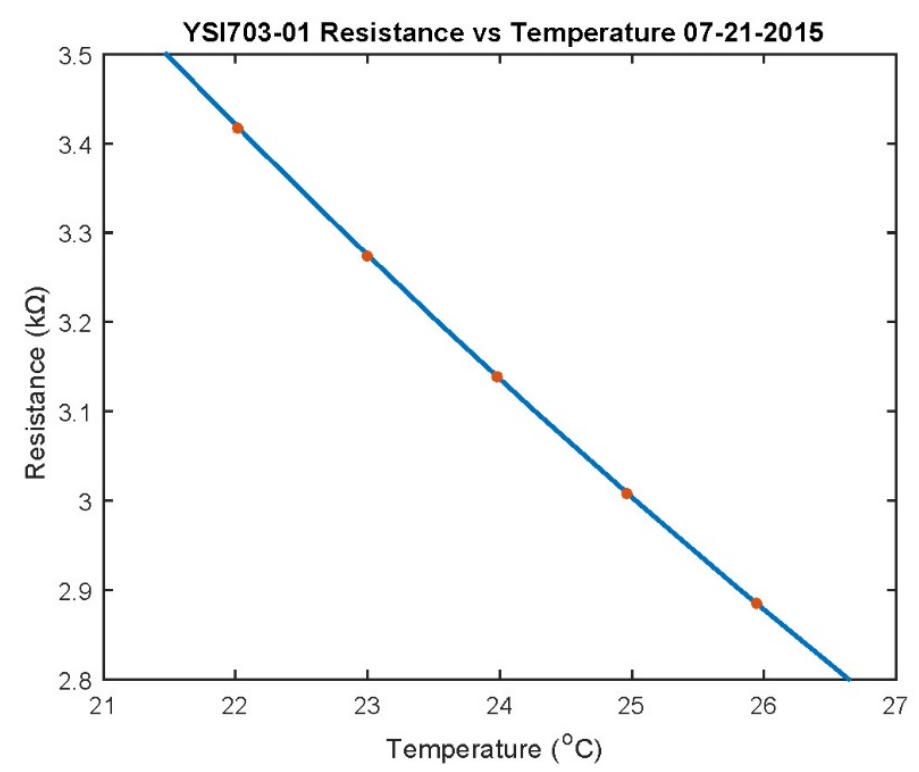

Fig. 5. An example of probe calibration. The red dots show the five calibration points, and the blue curve is the associated fit of the Steinhart-Hart equation.

\section{Uncertainty Analysis}

After calibration, the system is able to measure probe resistance and map it to temperature. The final step in the construction of this system was to find the uncertainty of these temperature measurements to determine whether the system could resolve the required $50 \mathrm{mK}$ range. Temperature measurements depend on four quantities: the three Steinhart-Hart coefficients, $A, B$, and $D$, and resistance $R$. To analyze this propagation of the error problem, we considered the following covariance matrix

$$
M_{c o v}=\left(\begin{array}{cccc}
\sigma_{A A} & \sigma_{A B} & \sigma_{A D} & 0 \\
\sigma_{B A} & \sigma_{B B} & \sigma_{B D} & 0 \\
\sigma_{D A} & \sigma_{D B} & \sigma_{D D} & 0 \\
0 & 0 & 0 & \sigma_{R r}
\end{array}\right),
$$

where the elements represent the covariance between the two quantities shown in subscript. The choice of a coefficient depends on the other coefficients and not $R$. Likewise, the measurement of $R$ does not depend on the coefficients. This is why all of the covariances with respect to $R$ are zero. To compute $\sigma_{k}$, the uncertainty of $k \equiv 1 / T$, we introduce the vector

$$
D=\left(\begin{array}{llll}
\frac{\partial k}{\partial A} & \frac{\partial k}{\partial B} & \frac{\partial k}{\partial D} & \frac{\partial k}{\partial R}
\end{array}\right)
$$

This allows us to write:

$$
\sigma_{k}^{2}=D M_{\mathrm{cov}} D^{T}
$$

Now, applying standard error propagation, we find that

$$
\sigma_{T_{\mathrm{cal}}}^{2}=\left(\frac{\partial T}{\partial k}\right)^{2} \sigma_{k}^{2}
$$

and

$$
\sigma_{T_{\text {cal }}}=\sigma_{k} T^{2}
$$


where $\sigma_{T \text { cal }}$ is the calibration uncertainty in $T$. For a complete uncertainty, we must also consider the error introduced by the temperature standard used in the calibration. Therefore, the total uncertainty $\sigma_{\mathrm{T}}$ is given by

$$
\sigma_{\mathrm{T}}=\sqrt{\sigma_{T_{\text {cal }}}^{2}+\sigma_{\text {probe }}^{2}}
$$

Figure 6 shows the results of this uncertainty analysis at each of the five calibration points for one of the probes. Across many calibrations, we found that $\sigma_{\mathrm{T}}$ is largest for the calibration at $22{ }^{\circ} \mathrm{C}$ and $26{ }^{\circ} \mathrm{C}$, and at the points of primary interest, $23^{\circ} \mathrm{C}$ and $25^{\circ} \mathrm{C}, \sigma_{\mathrm{T}}<9 \mathrm{mK}$ expanded uncertainty $(k=2)$ [8].

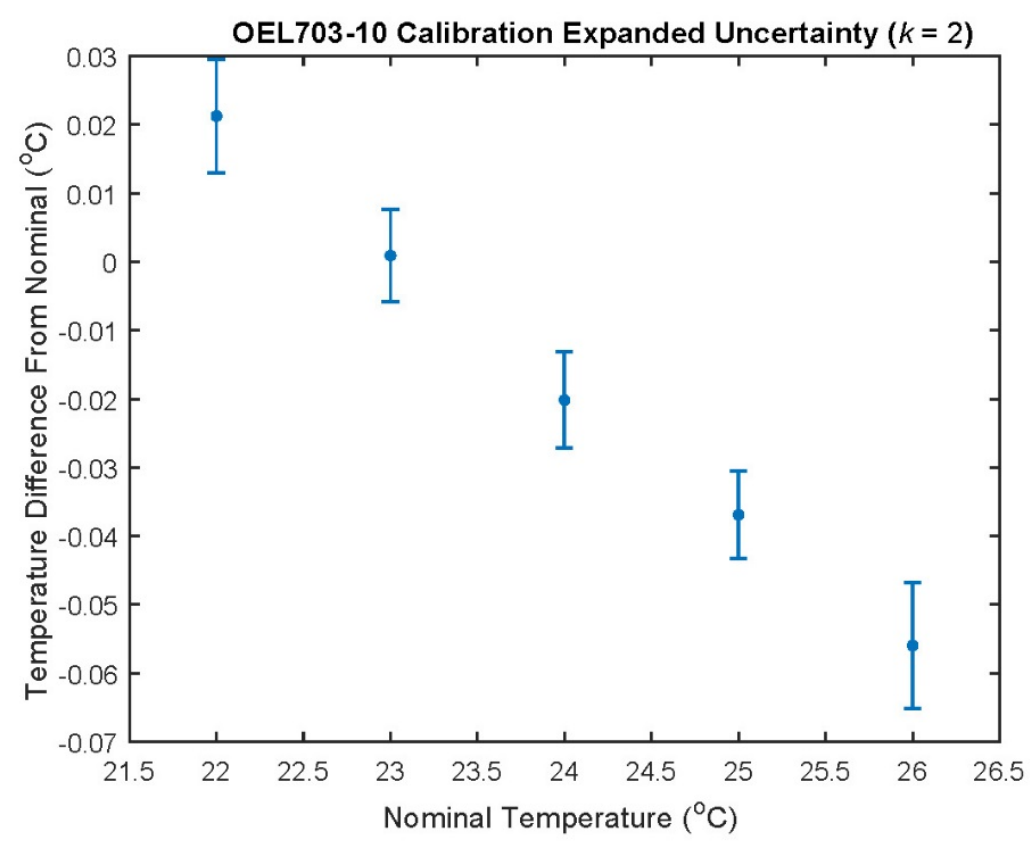

Fig. 6. The error bars show an expanded uncertainty $(k=2)$ at each of the calibration points. The uncertainty increases with the distance from the nominal set point due to instability in the oil bath temperature controller.

\section{Software}

The control systems for both temperature measurement and calibration were written in LabVIEW. The program first interfaces with the previously described instrumentation via a GPIB bus. When the system is being used for temperature monitoring after measuring the resistance of a probe, the temperature is calculated using the probe's Steinhart-Hart coefficients. This value is then displayed and saved to a secure networked drive. If the temperature measured is outside the allowed temperature range for the probe's location, an alarm will sound over the computer's speakers, and an email notification will be sent to all members of the project. The system also supports an additional list of facility services email addresses. In the case where a probe measuring the ambient temperature of the laboratory indicates that the laboratory temperature has drifted outside its specified range, facility services and laboratory personnel will be alerted to the problem. The frequency at which emails are sent has been limited to one per hour.

When the system is being used to calibrate probes, in addition to measuring resistance, the temperature of the reference probe is recorded. These values along with their standard deviations are written to file. Once the calibration measurements have been completed, a Python script performs the linear least squares fit and covariance matrix computations to generate a report including the Steinhart-Hart coefficients and uncertainty in a temperature measurement at each of the calibration points. 
Figure 7 shows part of the interface for the temperature monitoring system. The fields on the left side are for general system information, including the GPIB addresses of the DVM and scanner, temperature log file location, resistance and channel of the reference resistor, and email lists for laboratory and facility services recipients. Each probe channel has its own control panel with two tabs. The front tab (shown for channels 1 and 2) has controls allowing the user to turn the channel off, indicate the probe's location, input the allowed temperature range of this location, specify if the probe is measuring a bath or laboratory temperature, and mute its audio alarm. The last temperature recorded from the probe is also displayed on this tab along with two status indicators, one indicating the probe is currently being measured and the other indicating if the probe is within its desired range. The back tab (shown for channel 3) is for the calibration parameters, and it is where a probe's serial number and Steinhart-Hart coefficients can be entered. Channels 4 through 20 (not shown) fill the remainder of the screen to complete the interface.

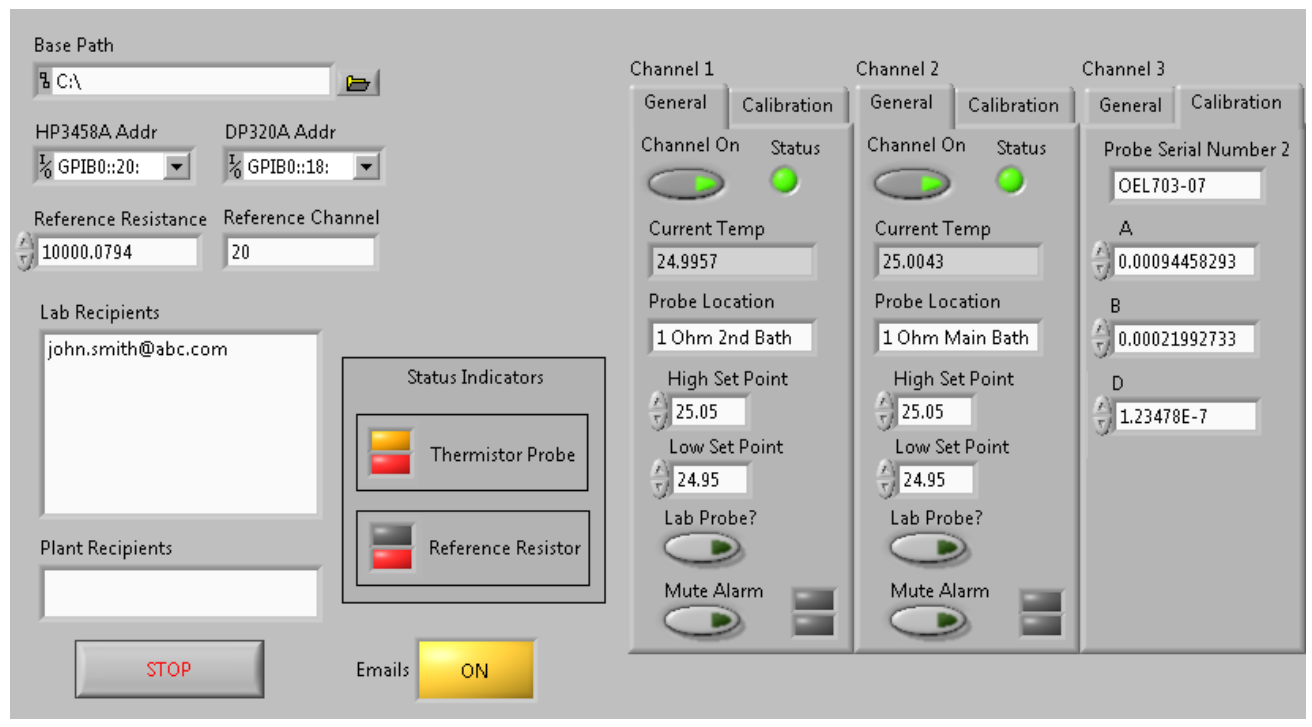

Fig. 7. The graphic user interface of the temperature monitoring system. Three of the twenty channels are shown.

Since initial deployment, only minor problems in its operation have occurred. The most notable of these was an intermittent issue with the scanner. It was discovered that the scanner would occasionally fail to latch, resulting in an error in the voltage measurement. This bug was eliminated with a software patch that used an error handling routine to ensure that relays were latched.

\section{Conclusion}

A temperature monitoring system was built and installed that continuously operates, monitors temperature in up to 19 locations, logs temperature data for each location, measures temperature with an expanded uncertainty $(k=2)$ of less than $9 \mathrm{mK}$, and has the function of sending email alerts when temperature limits are exceeded. With the addition of a reference thermometer and an oil bath, the system can also be used to calibrate the thermistor probes that measure temperature during normal operation. The Steinhart-Hart coefficients have been determined to characterize individual probes for resistance as a function of temperature. Voltage measurements are used to determine the probe resistance, which is then converted to temperature using the Steinhart-Hart coefficients. The uncertainty of the calibrated probes has been determined using a covariance matrix to evaluate the standard error of propagation with an expanded uncertainty $(k=2)$ of less than $9 \mathrm{mK}$ for the temperature range $22{ }^{\circ} \mathrm{C}$ to $26^{\circ} \mathrm{C}$. A Python script performs the linear least squares analysis, and for each probe calibration, it generates a test report that includes the Steinhart-Hart coefficients and uncertainty for each calibration temperature. During its 27 months of continuous operation, the system behaved appropriately during a planned HVAC outage in the building and successfully identified several failures of bath temperature controllers and power supplies, allowing timely 
corrective action to be taken by NIST staff. Besides resistance laboratories, this system has applications in other laboratory and industrial environments, where continuous temperature monitoring and automated alarms in multiple locations are required.

\section{Acknowledgments}

We would like to thank Mr. Gregory Strouse for his guidance regarding measurement technique and Dr. Stephan Schlamminger for his assistance with the uncertainty analysis. This work was support by NIST and National Science Foundation grant number 70NANB15H153.

\section{References}

[1] Elmquist R, Jarrett D, Jones G, Kraft M, Shields S, Dziuba R (2003) NIST Measurement Service for DC Standard Resistors. U.S. Department of Commerce, Washington, D.C., NIST Technical Note 1458. https://doi.org/10.6028/NIST.TN.1458.

[2] Jones G, Elmquist R (2007) Temperature and pressure coefficients of resistance for Thomas $1 \Omega$ resistors. Measure 2(2):42-48. https://doi.org/10.1080/19315775.2007.11721371.

[3] Moore J, Davis C, Coplan M (2015) Building Scientific Apparatus (Cambridge University Press, Cambridge, UK), 4th Ed., p 607.

[4] Vaughn C, Gartenhaus J, Strouse G (2006) NIST calibration uncertainties of thermistor thermometers over the range from $-50^{\circ} \mathrm{C}$ to $90{ }^{\circ} \mathrm{C}$. Measure 1(2):36-41. https://doi.org/10.1080/19315775.2006.11721320.

[5] Maxim Integrated Products (1996) Phase-reversal analog switches. Maxim Integrated Products, San Jose, CA.

[6] Steinhart J, Hart S (1968) Calibration curves for thermistors. Deep Sea Research and Oceanographic Abstracts 15(4):497-503. https://doi.org/10.1016/0011-7471(68)90057-0.

[7] White DR, Hill K, del Campo D, Garcia Izquierdo C (2014) Guide on Secondary Thermometry: Thermistor Thermometry. BIPM, Sevres, France. Available at: http://www.bipm.org/utils/common/pdf/ITS-90/Guide-SecTh-Thermistor-Thermometry.pdf.

[8] Taylor J (1997) An Introduction to Error Analysis: The Study of Uncertainties in Physical Measurement (University Science Books, Sausalito, CA), 2nd Ed.

About the authors: Daniel Paseltiner was a Summer Undergraduate Research Fellow (SURF) in the Fundamental Electrical Measurements Group of the Quantum Measurement Division of NIST in the summer of 2015. Currently, he works as research assistant and programmer in a neuroscience laboratory at Bates College.

Shamith Payagala is an electrical engineer in the Fundamental Electrical Measurements Group of the Quantum Measurement Division of NIST.

Dean Jarrett is an electrical engineer in the Fundamental Electrical Measurements Group of the Quantum Measurement Division of NIST as well. He leads the Metrology of the Ohm Project, and highresistance standards and measurement techniques are his main area of research.

The National Institute of Standards and Technology is an agency of the U.S. Department of Commerce. 